MITSUBISHI ELECTRIC RESEARCH LABORATORIES

http://www.merl.com

\title{
Integrated Design of Speed Sensorless Control Algorithms for Induction Motors
}

\author{
Zhang, J.; Wang, Y.; Bortoff, S.A.; Satake, A.; Furutani, S.; Deng, X. \\ TR2015-093 July 30, 2015
}

\begin{abstract}
Bandwidth limitation hinders the economical and broad application of induction motors promised by speed sensorless drive. This paper proposes an integrated control and observer design for speed sensorless control of induction motors with stator voltage and current measurements. With a general observer structure, the backstepping-based robust control design explicitly considers the errors of both the state estimation and tracking, and thus avoids the commonly used time scale separation. The gain selection for the controller becomes easy and straightforward. As a case study, the extended Kalman filter is used as a state estimator in order to simplify the gain tuning process. Simulation results validate the effectiveness of proposed method.
\end{abstract}

2015 Chinese Control Conference (CCC) \& SICE Annual Conference

This work may not be copied or reproduced in whole or in part for any commercial purpose. Permission to copy in whole or in part without payment of fee is granted for nonprofit educational and research purposes provided that all such whole or partial copies include the following: a notice that such copying is by permission of Mitsubishi Electric Research Laboratories, Inc.; an acknowledgment of the authors and individual contributions to the work; and all applicable portions of the copyright notice. Copying, reproduction, or republishing for any other purpose shall require a license with payment of fee to Mitsubishi Electric Research Laboratories, Inc. All rights reserved. 



\title{
Integrated Design of Speed Sensorless Control Algorithms for Induction Motors
}

\author{
Jian Zhang ${ }^{1}$, Yebin Wang ${ }^{2}$, Scott A. Bortoff ${ }^{2}$, Akira Satake ${ }^{3}$, Shinichi Furutani ${ }^{3}$, Xinyan Deng ${ }^{1}$, Bin Yao ${ }^{1}$ \\ 1. School of Mechanical Engineering, Purdue University, 585 Purdue Mall, West Lafayette, IN 47907 USA. \\ E-mail: zhang699@purdue.edu, xdeng@purdue.edu \\ 2. Mitsubishi Electric Research Laboratories, 201 Broadway, Cambridge, MA 02139 USA. \\ E-mail: yebinwang@ieee.org, bortoff@merl.com \\ 3. the Advanced Technology R\&D Center, Mitsubishi Electric Corporation, 8-1-1, Tsukaguchi-honmachi, Amagasaki City, 661-8661, \\ Japan. \\ E-mail: Satake.Akira@dy.MitsubishiElectric.co.jp, Furutani.Shinichi@dw.MitsubishiElectric.co.jp
}

\begin{abstract}
Bandwidth limitation hinders the economical and broad application of induction motors promised by speed sensorless drive. This paper proposes an integrated control and observer design for speed sensorless control of induction motors with stator voltage and current measurements. With a general observer structure, the backstepping-based robust control design explicitly considers the errors of both the state estimation and tracking, and thus avoids the commonly used time scale separation. The gain selection for the controller becomes easy and straightforward. As a case study, the extended Kalman filter is used as a state estimator in order to simplify the gain tuning process. Simulation results validate the effectiveness of proposed method.
\end{abstract}

Key Words: Speed sensorless control, induction motor, extended Kalman filter, backstepping

\section{Introduction}

Speed regulation of induction motors is an old but interesting problem. Techniques to achieve the speed regulation have evolved from variable frequency control to vector control and its variants for instance direct/indirect field-oriented state feedback control, speed-sensorless control [1,2], adaptive field-oriented control $[1,3]$. The vector control with full state or the rotor speed measurements results in good performance at expenses of extra sensors, and thus limits its application in practice. Recent work is devoted to speed sensorless control algorithms, e.g. without the speed measurement.

Speed-sensorless control design problem is practically meaningful and challenging, and thus attracts a lot of theoretical interests, e.g. [4,5]. Adaptive idea, where the rotor speed is typically treated as an unknown parameter to avoid nonlinearity in dynamics, was initially exploited and is still prevailing in the speed-sensorless motor drives [6-10]. Designs relying on the assumption of the speed as a constant/slow-varying parameter suffer unsatisfactory transient performance inherent to adaptation. $\mathrm{Nu}$ merous work tried to avoid the speed-as-parameter assumption for instance high gain observer [2], sliding mode observer [11-13], and extended kalman filter (EKF) [14] etc., but failed to address the drive performance aspect. As an example, resorting to nonlinear observer design techniques entails the system in certain normal forms, which turns out to be difficult. Well-known high gain observer design assumes observable form (OF). The induction motor model under any frame is hardly put into OF due to the complexity of the state transformation and difficulty to compute the inverse transformation. Various work circumvented the problem by considering open-loop flux observer, e.g. [2], thus lead to drives with slow responses.

This paper proposes an integrated control and observer design framework for speed sensorless control of induction

This work was done while J. Zhang was an intern with Mitsubishi Electric Research Laboratories, 201 Broadway, Cambridge, MA 02139 USA. motors with stator current measurements. With a general observer structure, the backstepping-based robust control design explicitly considers all the state estimation and tracking errors. The gain selection for the controller is straightforward. On the state estimation side, as a case study, an extended Kalman filter is designed to simplify the gain tuning process. Simulation results are presented to show the following facts: 1) the proposed method can achieve high bandwidth and high precision speed sensorless tracking control of a typical induction motor under practical constraints $(200 \mathrm{~Hz}$ control bandwidth for $20 \mathrm{KHz}$ sampling rate); 2) the gain selection for the proposed method is systematic and simple; 3 ) the proposed method can track various trajectories as long as the state and control input constraints are considered. No dependence of controller and/or observer on the trajectory is found.

\section{Preliminaries}

\subsection{The Induction Motor Model}

With the assumption of linear magnetic circuits and balanced operating conditions, the two-phase equivalent model of an induction motor, represented in the fixed $a-b$ reference frame, can be written as follows [15],

$$
\begin{aligned}
\dot{\omega} & =\frac{3}{2} \frac{n_{p} M}{J L_{r}}\left(\phi_{r a} i_{s b}-\phi_{r b} i_{s a}\right)-T_{L} / J \\
\dot{i}_{s a} & =\frac{M R_{r}}{\sigma L_{r}^{2}} \phi_{r a}+\frac{n_{p} M}{\sigma L_{r}} \omega \phi_{r b}-\gamma i_{s a}+\frac{1}{\sigma} u_{s a}, \\
\dot{i}_{s b} & =\frac{M R_{r}}{\sigma L_{r}^{2}} \phi_{r b}-\frac{n_{p} M}{\sigma L_{r}} \omega \phi_{r a}-\gamma i_{s b}+\frac{1}{\sigma} u_{s b}, \\
\dot{\phi}_{r a} & =-\frac{R_{r}}{L_{r}} \phi_{r a}-n_{p} \omega \phi_{r b}+\frac{R_{r}}{L_{r}} M i_{s a}, \\
\dot{\phi}_{r b} & =-\frac{R_{r}}{L_{r}} \phi_{r b}+n_{p} \omega \phi_{r a}+\frac{R_{r}}{L_{r}} M i_{s b}, \\
y & =\left[i_{s a}, i_{s b}\right]^{T}
\end{aligned}
$$

where the subscripts $r$ and $s$ stand for the stator and the rotor, respectively; the subscripts $a$ and $b$ denote the $a$ and $b$ axis, 
respectively; $\omega$ is the angular speed of the rotor, $i$ denotes the current, and $\phi$ denotes the flux linkage; $u$ is the stator voltage input; $R, L, M, J, T_{L}$ and $n_{p}$ denote the resistance, inductance, mutual inductance, rotor inertia, load torque, and number of pole pairs, respectively. For compact notations, following lumped parameters are introduced with $\alpha=\frac{R_{r}}{L_{r}}$, $\beta=\frac{M}{\sigma L_{r}}, \sigma=L_{s}-\frac{M^{2}}{L_{r}}, \gamma=\frac{M^{2} R_{r}+L_{r}^{2} R_{s}}{\sigma L_{r}^{2}}=\frac{R_{s}}{\sigma}+\alpha \beta M$, and $\mu=\frac{3}{2} \frac{n_{p} M}{J L_{r}}$. We assume that the load torque $T_{L}$ can be parameterized as $T_{L}(\omega)=T_{0}+C_{f} \omega$ with $T_{0}$ an unknown constant. With the following change of notations, $x_{1}=\omega$, $x_{2}=i_{s a}, x_{3}=i_{s b}, x_{4}=\phi_{r a}, x_{5}=\phi_{r b}, u_{1}=u_{s a}$, $u_{2}=u_{s b}, b=\frac{1}{\sigma}, a_{1}=\mu, a_{2}=\frac{T_{0}}{J}, a_{3}=\frac{C_{f}}{J}, a_{5}=\alpha \beta$, $a_{6}=n_{p} \beta, a_{7}=\gamma, a_{8}=\alpha, a_{9}=n_{p}$, and $a_{10}=\alpha M$, one can rewrite the induction model (1) as

$$
\begin{aligned}
\dot{x} & =f(x, u)=g(x)+B u, \\
y= & C x, \\
g(x)= & {\left[\begin{array}{c}
a_{1}\left(x_{3} x_{4}-x_{2} x_{5}\right)-a_{2}-a_{3} x_{1} \\
a_{5} x_{4}+a_{6} x_{1} x_{5}-a_{7} x_{2} \\
a_{5} x_{5}-a_{6} x_{1} x_{4}-a_{7} x_{3} \\
-a_{8} x_{4}-a_{9} x_{1} x_{5}+a_{10} x_{2} \\
-a_{8} x_{5}+a_{9} x_{1} x_{4}+a_{10} x_{3}
\end{array}\right], } \\
B= & {\left[\begin{array}{ll}
0 & 0 \\
b & 0 \\
0 & b \\
0 & 0 \\
0 & 0
\end{array}\right], \quad C=\left[\begin{array}{lllll}
0 & 1 & 0 & 0 & 0 \\
0 & 0 & 1 & 0 & 0
\end{array}\right], }
\end{aligned}
$$

with $x=\left[x_{1}, x_{2}, x_{3}, x_{4}, x_{5}\right]^{T}$ and $u=\left[u_{1}, u_{2}\right]^{T}$.

\subsection{Problem Formulation}

General specifications for speed-sensorless electric drives using vector control is to regulate two variables: the rotor speed and the rotor flux magnitude $[1,2]$ given by

$$
\left[\begin{array}{c}
x_{1} \\
x_{4}^{2}+x_{5}^{2}
\end{array}\right]
$$

More specifically, the problem considered in this work is: Given the IM model (2), synthesize control inputs $u_{1}$ and $u_{2}$ such that $x_{1}$ and $x_{4}^{2}+x_{5}^{2}$ track $\omega_{d}(t)$ and $\psi_{d}^{2}$, respectively.

\section{Main Results: Observer Design}

\subsection{A General Observer}

For the integrated design of observer and controller, a general observer structure is first assumed as $\dot{\hat{x}}_{i}=\hat{f}_{i}+s i_{i}, 1 \leq$ $i \leq 5$, i.e.,

$$
\begin{aligned}
& \dot{\hat{x}}_{1}=a_{1}\left(x_{3} \hat{x}_{4}-x_{2} \hat{x}_{5}\right)-\hat{a}_{2}-a_{3} \hat{x}_{1}+s i_{1}\left(\tilde{x}_{2}, \tilde{x}_{3}\right) \\
& \dot{\hat{x}}_{2}=a_{5} \hat{x}_{4}+a_{6} \hat{x}_{1} \hat{x}_{5}-a_{7} \hat{x}_{2}+b u_{1}+s i_{2}\left(\tilde{x}_{2}, \tilde{x}_{3}\right) \\
& \dot{\hat{x}}_{3}=a_{5} \hat{x}_{5}-a_{6} \hat{x}_{1} \hat{x}_{4}-a_{7} \hat{x}_{3}+b u_{2}+s i_{3}\left(\tilde{x}_{2}, \tilde{x}_{3}\right) \\
& \dot{\hat{x}}_{4}=-a_{8} \hat{x}_{4}-a_{9} \hat{x}_{1} \hat{x}_{5}+a_{10} \hat{x}_{2}+s i_{4}\left(\tilde{x}_{2}, \tilde{x}_{3}\right) \\
& \dot{\hat{x}}_{5}=-a_{8} \hat{x}_{5}+a_{9} \hat{x}_{1} \hat{x}_{4}+a_{10} \hat{x}_{3}+s i_{5}\left(\tilde{x}_{2}, \tilde{x}_{3}\right)
\end{aligned}
$$

For continuous case, such as Extended Kalman Filter (EKF) and Luenberger observer:

$$
\begin{aligned}
& s_{1}\left(\tilde{x}_{2}, \tilde{x}_{3}\right)=l_{11} \tilde{x}_{2}+l_{12} \tilde{x}_{3} \\
& \operatorname{si}_{2}\left(\tilde{x}_{2}, \tilde{x}_{3}\right)=l_{21} \tilde{x}_{2}+l_{22} \tilde{x}_{3} \\
& s i_{3}\left(\tilde{x}_{2}, \tilde{x}_{3}\right)=l_{31} \tilde{x}_{2}+l_{32} \tilde{x}_{3} \\
& s_{4}\left(\tilde{x}_{2}, \tilde{x}_{3}\right)=l_{41} \tilde{x}_{2}+l_{42} \tilde{x}_{3} \\
& \operatorname{si}_{5}\left(\tilde{x}_{2}, \tilde{x}_{3}\right)=l_{51} \tilde{x}_{2}+l_{52} \tilde{x}_{3},
\end{aligned}
$$

where $L$ is the matrix form of the observer gains $l_{11}, \ldots$, $l_{52}$. For discontinuous case, such as sliding mode observer (SMO):

$$
\begin{aligned}
& s_{1}\left(\tilde{x}_{2}, \tilde{x}_{3}\right)=h_{11} S\left(\tilde{x}_{2}\right)+h_{12} S\left(\tilde{x}_{3}\right) \\
& \operatorname{si}_{2}\left(\tilde{x}_{2}, \tilde{x}_{3}\right)=h_{21} S\left(\tilde{x}_{2}\right)+h_{22} S\left(\tilde{x}_{3}\right) \\
& s_{3}\left(\tilde{x}_{2}, \tilde{x}_{3}\right)=h_{31} S\left(\tilde{x}_{2}\right)+h_{32} S\left(\tilde{x}_{3}\right) \\
& \sin _{4}\left(\tilde{x}_{2}, \tilde{x}_{3}\right)=h_{41} S\left(\tilde{x}_{2}\right)+h_{42} S\left(\tilde{x}_{3}\right) \\
& \sin _{5}\left(\tilde{x}_{2}, \tilde{x}_{3}\right)=h_{51} S\left(\tilde{x}_{2}\right)+h_{52} S\left(\tilde{x}_{3}\right) .
\end{aligned}
$$

Switching, however, induces chattering and thus continuous approximations are often adopted instead: $S\left(\tilde{x}_{2}\right) \approx l_{2 \text { eff }} \tilde{x}_{2}$ and $S\left(\tilde{x}_{3}\right) \approx l_{3 e f f} \tilde{x}_{3}$. L can still be defined with effective gains $l_{2 e f f}$ and $l_{3 e f f}$.

Define estimation errors as $\tilde{x}=x-\hat{x}$. Using prior information of the original system states, one can construct a projection mapping to ensure the boundedness of state estimates:

$$
\begin{aligned}
& {\dot{\widehat{x_{i}}}}_{i}=\operatorname{Proj}_{\widehat{x}_{i}}\left(\tau_{i}\right) \\
& \tau_{i}=\hat{f}_{i}+s i_{i} \\
& \hat{f}_{i}=f_{i}\left(\hat{x}, \hat{a}_{2}\right) .
\end{aligned}
$$

The projection mapping of a scalar is defined in [16] as

$$
\operatorname{Proj}_{\widehat{x}_{i}}\left(\bullet_{i}\right)= \begin{cases}0 & \text { if } \widehat{x}_{i}=x_{\text {imax }} \text { and } \bullet_{i}>0 \\ 0 & \text { if } \widehat{x}_{i}=x_{\text {imin }} \text { and } \bullet_{i}<0 \\ \bullet_{i} & \text { otherwise }\end{cases}
$$

When applied to a vector $\widehat{x}$, the project mapping is component-wise, i.e., $\operatorname{Proj}_{\widehat{x}}(\bullet)=$ $\left[\operatorname{Proj}_{\widehat{x}_{1}}\left(\bullet_{1}\right), \ldots, \operatorname{Proj}_{\widehat{x}_{5}}\left(\bullet_{5}\right)\right]^{T}$. It can be shown that for any adaptation function $\tau$, the projection mapping used in (8) guarantees

(P1)

$$
\widehat{x} \in \Omega_{x} \triangleq\left\{\widehat{x}: x_{i \min } \leq \widehat{x} \leq x_{\text {imax }}\right\}
$$

A slowly varying load torque can be treated by augmenting the state $x$ with $a_{2}$ which will be adapted in the observer design. In addition, other slowly changing quantities, $R_{r}, a_{5}$ $a_{7} a_{8} a_{10}$, can also be treated as unknown parameters $\theta$ and extended for adaptation within the same design framework.

\subsection{Observer Design}

As a case study, the EKF is designed to simplify the gain tuning process as the Kalman gains are automatically adjusted along the state trajectory. With sampling rate of the filter as $T_{s}$ and forward difference method, the system dynamics (2) can be discretized as

$$
x_{k}=T_{s} f\left(x_{k-1}, u_{k-1}\right)+x_{k-1}=f^{\prime}\left(x_{k-1}, u_{k-1}\right)
$$


Linearization of the dynamics,

$$
A=\frac{\partial f}{\partial x}=\left[\begin{array}{ccccc}
-a_{3} & -a_{1} x_{5} & a_{1} x_{4} & a_{1} x_{3} & -a_{1} x_{2} \\
a_{6} x_{5} & -a_{7} & 0 & a_{5} & a_{6} x_{1} \\
-a_{6} x_{4} & 0 & -a_{7} & -a_{6} x_{1} & a_{5} \\
-a_{9} x_{5} & a_{10} & 0 & -a_{8} & -a_{9} x_{1} \\
a_{9} x_{4} & 0 & a_{10} & a_{9} x_{1} & -a_{8}
\end{array}\right]
$$

Thus

$$
A_{k}=T_{s} A+I
$$

\section{Predict:}

Predicted state estimate

$$
\hat{x}_{k \mid k-1}=f^{\prime}\left(\hat{x}_{k-1 \mid k-1}, u_{k \mid k-1}\right)
$$

Predicted covariance estimate

$$
P_{k \mid k-1}=A_{k-1} P_{k-1 \mid k-1} A_{k-1}^{T}+Q_{k-1}
$$

\section{Update:}

Measurement residual

$$
\tilde{y}_{k}=z_{k}-C \hat{x}_{k \mid k-1}
$$

Residual covariance

$$
S_{k}=C_{k} P_{k \mid k-1} C_{k}^{T}+R_{k}
$$

Near-optimal Kalman gain

$$
K_{k}=P_{k \mid k-1} C_{k}^{T} S_{k}^{-1}
$$

Updated state estimate

$$
\hat{x}_{k \mid k}=\hat{x}_{k \mid k-1}+K_{k} \tilde{y}_{k}
$$

Updated covariance estimate

$$
P_{k \mid k}=\left(I-K_{k} C_{k}\right) P_{k \mid k-1} .
$$

For general observer form

$$
L=\frac{K_{k}}{T_{s}}
$$

\section{Main Results: Integrated Control Design}

We consider the control design for the simplified case where a system suffers from uncertainties $\widetilde{d}$. For the induction motor case, uncertain nonlinearities could be result from the state estimation errors. The following practical assumption thus is required to establish stability results.

Assumption 4.1 Uncertain nonlinearities are bounded, i.e.,

$$
\widetilde{d} \in \Omega_{d} \triangleq\left\{\widetilde{d}:\|\widetilde{d}\| \leq \delta_{d}\right\}
$$

where $\widetilde{d}=d-d_{n}$, and $\delta_{d}$ is also a known bounding function.

With (P1) in (9), the state observer can guarantee the bounded state estimation error. The following assumption can be made for the control design,
Assumption 4.2 The bounds of state estimation errors are known, i.e.,

$$
\begin{gathered}
\tilde{x} \in \Omega_{\tilde{x}} \triangleq\left\{\tilde{x}: \tilde{x}_{\min } \leq \tilde{x} \leq \tilde{x}_{\max }\right\} \\
\text { where } \tilde{x}_{\text {min }}=\left[\tilde{x}_{1 \min }, \ldots, \tilde{x}_{5 \min }\right]^{T} \text { and } \tilde{x}_{\max }= \\
{\left[\tilde{x}_{1 \max }, \ldots, \tilde{x}_{5 \max }\right]^{T} \text { are known. }}
\end{gathered}
$$

The control design consists of four steps. Step 1 is the speed control loop with electromagnetic torque as an virtual control; step 2 is a robust torque control loop which regulates the electromagnetic torque from the speed loop in the first step; step 3 is the flux tracking loop which regulates the magnetic flux magnitude for efficient operation; step 4 delivers the required virtual control from step 3 using a similar robust control design. State estimation errors are explicitly taken into account within all control design steps as bounded model uncertainties. The controller and observer structure are illustrated in Fig. 1 with notations to be introduced in the following design steps.

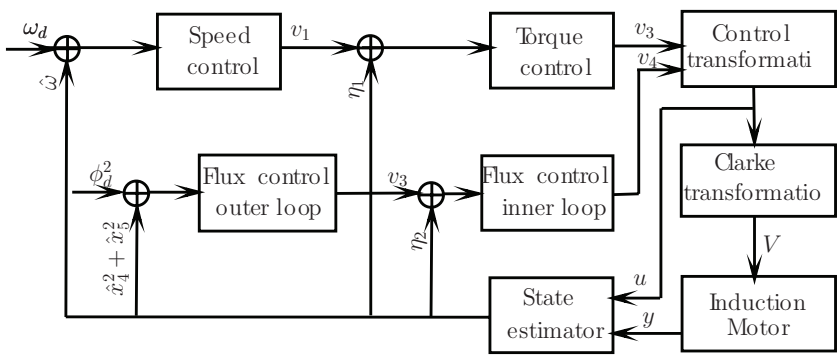

Fig. 1: Controller and observer structure.

\subsection{Step 1-Speed Loop Control Design}

Define the speed tracking error $z_{1}=\hat{x}_{1}-\omega_{d}$, and compute its time derivative

$$
\begin{aligned}
\dot{z}_{1}= & -a_{3} z_{1}+a_{1}\left(x_{3} \hat{x}_{4}-x_{2} \hat{x}_{5}\right)+s i_{1}\left(\tilde{x}_{2}, \tilde{x}_{3}\right) \\
& -\dot{\omega}_{d}-a_{3} \omega_{d}-\hat{a}_{2} .
\end{aligned}
$$

Introduce a virtue control $v_{1}$, and define the discrepancy between $v_{1}$ and the estimated electromagnetic torque $a_{1}\left(x_{3} \hat{x}_{4}-x_{2} \hat{x}_{5}\right)$ as follows

$$
z_{2}=a_{1}\left(x_{3} \hat{x}_{4}-x_{2} \hat{x}_{5}\right)-v_{1} .
$$

Thus the speed tracking error can be rewritten as

$$
\dot{z}_{1}=-a_{3} z_{1}+v_{1}+z_{2}+s i_{1}\left(\tilde{x}_{2}, \tilde{x}_{3}\right)-\dot{\omega}_{d}-a_{3} \omega_{d}-\hat{a}_{2} .
$$

The virtual control $v_{1}$ is split into two parts

$$
v_{1}=v_{1 a}+v_{1 s},
$$

where $v_{1 a}$ is the feedforward model compensation given by

$$
v_{1 a}=-s i_{1}\left(\tilde{x}_{2}, \tilde{x}_{3}\right)+\dot{\omega}_{d}+a_{3} \omega_{d}+\hat{a}_{2},
$$

and $v_{1 s}$ is the feedback stabilization control

$$
v_{1 s}=-k_{1 s 1} z_{1} .
$$

Thus (25) can be rewritten as follows

$$
\dot{z}_{1}=-k_{1} z_{1}+z_{2}, \quad k_{1}=a_{3}+k_{1 s 1} .
$$


Overall the virtual control $v_{1}$ is given by

$$
v_{1}=-k_{1 s 1} z_{1}-s i_{1}\left(\tilde{x}_{2}, \tilde{x}_{3}\right)+\dot{\omega}_{d}+a_{3} \omega_{d}+\hat{a}_{2},
$$

and its derivative is computed as follows

$$
\begin{aligned}
\dot{v}_{1}= & k_{1 s 1} k_{1} z_{1}+\ddot{\omega}_{d}+a_{3} \omega_{d}+\hat{a}_{2} \\
& +\left(l_{11} a_{7}+l_{11} l_{21}+l_{12} l_{31}\right) \tilde{x}_{2} \\
& +\left(l_{12} a_{7}+l_{11} l_{22}+l_{12} l_{32}\right) \tilde{x}_{3} \\
& -\left(l_{11} a_{5}-l_{12} a_{6} \hat{x}_{1}\right) \tilde{x}_{4}-\left(l_{12} a_{5}+l_{11} a_{6} \hat{x}_{1}\right) \tilde{x}_{5} \\
& -\left(l_{11} a_{6} x_{5}-l_{12} a_{6} x_{4}\right) \tilde{x}_{1}-k_{1 s 1} z_{2},
\end{aligned}
$$

which will be used in the second step.

\subsection{Step 2-Robust Torque Control Design}

According to (24), (31), and (4), we have

$$
\dot{z}_{2}=v_{2}+\psi_{2}+\tilde{d}_{2}
$$

where $v_{2}=a_{21} b u_{1}+a_{22} b u_{2}$ with $a_{21}=-a_{1} \hat{x}_{5}, a_{22}=$ $a_{1} \hat{x}_{4}$, and

$$
\begin{aligned}
\psi_{2}= & -\left(a_{7}+a_{8}\right) v_{1}-a_{1} a_{6} \hat{x}_{1}\left(\hat{x}_{4}^{2}+\hat{x}_{5}^{2}\right) \\
& -a_{1} a_{9} \hat{x}_{1}\left(\hat{x}_{5} x_{3}+\hat{x}_{4} x_{2}\right) \\
& +a_{1} a_{10}\left(\hat{x}_{2} x_{3}-\hat{x}_{3} x_{2}\right)+a_{1} x_{3} s i_{4}\left(\tilde{x}_{2}, \tilde{x}_{3}\right) \\
& -a_{1} x_{2} s i_{5}\left(\tilde{x}_{2}, \tilde{x}_{3}\right)-k_{1 s 1} \mathrm{k}_{1} z_{1}-\ddot{\omega}_{d}-a_{3} \dot{\omega}_{d} \\
& -\dot{\hat{a}}_{2}-\left(l_{11} a_{7}+l_{11} l_{21}+l_{12} l_{31}\right) \tilde{x}_{2} \\
& -\left(l_{12} a_{7}+l_{11} l_{22}+l_{12} l_{32}\right) \tilde{x}_{3}+k_{1 s 1} z_{2} \\
\tilde{d}_{2}= & a_{1} a_{5}\left(\tilde{x}_{5} \hat{x}_{4}-\tilde{x}_{4} \hat{x}_{5}\right)-a_{1} a_{6} x_{1}\left(\tilde{x}_{4} \hat{x}_{4}+\tilde{x}_{5} \hat{x}_{5}\right) \\
& -a_{1} a_{6} \tilde{x}_{1}\left(\hat{x}_{4}^{2}+\hat{x}_{5}^{2}\right)+\left(l_{11} a_{5}-l_{12} a_{6} \hat{x}_{1}\right) \tilde{x}_{4} \\
& +\left(l_{12} a_{5}+l_{11} a_{6} \hat{x}_{1}\right) \tilde{x}_{5} \\
& +\left(l_{11} a_{6} x_{5}-l_{12} a_{6} x_{4}\right) \tilde{x}_{1},
\end{aligned}
$$

where $\tilde{d}_{2}$ denotes the disturbance due to state estimation errors. One can try to estimate it which may result in improved system performance or reject it using the prior knowledge of its bounds. We here treat it as a bounded disturbance. The control $v_{2}$ is rewritten as follows

$$
v_{2}=v_{2 a}+v_{2 s},
$$

where the feedforward model compensation term is

$$
v_{2 a}=-\psi_{2}-\tilde{d}_{2}-z_{1}
$$

and the feedback stabilization term $v_{2 s}$ consists of the nominal stabilization term $v_{2 s 1}$ and the robust control term $v_{2 s 2}$

$$
\begin{aligned}
& v_{2 s}=v_{2 s 1}+v_{2 s 2}, \\
& v_{2 s 1}=-k_{2 s 1} z_{2}, \\
& k_{2}=a_{7}+a_{8}+k_{2 s 1} .
\end{aligned}
$$

Thus (32) can be rearranged as follows

$$
\dot{z}_{2}=-k_{2} z_{2}+v_{2 s 2}+\tilde{d}_{2}-z_{1} .
$$

Given Assumption 4.2, $\tilde{d}_{2}$ is bounded. Thus there exists $v_{2 s 2}$ such that the following conditions hold

- $z_{2}\left(v_{2 s 2}+\tilde{d}_{2}\right) \leq \epsilon_{2}$,

- $z_{2} v_{2 s 2} \leq 0$, where $\epsilon_{2}$ is a design parameter and can be arbitrarily small. One example of $v_{2 s 2}$ that satisfies above conditions can be taken as follows

$$
v_{2 s 2}=-\frac{1}{4 \epsilon_{2}} h_{2}^{2} z_{2}
$$

where $h_{2}=\delta_{d_{2}}$ and $\delta_{d_{2}}$ is the bound of $\tilde{d}_{2}$.

Remark 4.3 The aforementioned control $v_{1}$ and $v_{2}$ ensures that all signals are bounded. We define a positive definite function

$$
V_{s 2}=\frac{1}{2} z_{1}^{2}+\frac{1}{2} z_{2}^{2}
$$

and have its time derivative

$$
\begin{aligned}
\dot{V}_{s 2} & =-k_{1} z_{1}^{2}+z_{1} z_{2}+z_{2} \dot{z}_{2} \\
& =-k_{1} z_{1}^{2}-k_{2} z_{2}^{2}+z_{2}\left(v_{2 s 2}+\tilde{d}_{2}\right) \\
& \leq-k_{1} z_{1}^{2}-k_{2} z_{2}^{2}+\epsilon_{2} \\
& \leq-\lambda_{2} V_{s 2}+\epsilon_{2} .
\end{aligned}
$$

Hence, $V_{s 2}$ is bounded above by

$$
V_{s 2} \leq \exp \left(-\lambda_{2} t\right) V_{s 2}(0)+\frac{\epsilon_{2}}{\lambda_{2}}\left[1-\exp \left(-\lambda_{2} t\right)\right],
$$

where $\lambda_{2}=2 \min \left(k_{1}, k_{2}\right)$.

\subsection{Step 3-Flux Outer Loop Control Design}

Flux tracking control is designed using backstepping. Define the flux modules tracking error

$$
z_{3}=\hat{x}_{4}^{2}+\hat{x}_{5}^{2}-\psi_{d}
$$

and compute its time derivative

$$
\dot{z}_{3}=2 a_{10}\left(x_{2} \hat{x}_{4}+x_{3} \hat{x}_{5}\right)+\psi_{3},
$$

where

$$
\begin{aligned}
\psi_{3}= & -2 a_{8}\left(\hat{x}_{4}^{2}+\hat{x}_{5}^{2}\right)-2 a_{10}\left(\tilde{x}_{2} \hat{x}_{4}+\tilde{x}_{3} \hat{x}_{5}\right) \\
& +2 l_{41} \tilde{x}_{2} \hat{x}_{4}+2 l_{42} \tilde{x}_{3} \hat{x}_{4}+2 l_{51} \tilde{x}_{2} \hat{x}_{5}+2 l_{52} \tilde{x}_{3} \hat{x}_{5}-\dot{\psi}_{d} .
\end{aligned}
$$

Note that $\psi_{3}$ depends on accessible signals. Introduce a virtual control $v_{3}$, and a state $z_{4}$ to denote the discrepancy between $v_{3}$ and $2 a_{10}\left(x_{2} \hat{x}_{4}+x_{3} \hat{x}_{5}\right)$, i.e.,

$$
z_{4}=2 a_{10}\left(x_{2} \hat{x}_{4}+x_{3} \hat{x}_{5}\right)-v_{3} .
$$

Similar to $v_{1}$, the virtual control $v_{3}$ is rewritten as

$$
v_{3}=v_{3 a}+v_{3 s},
$$

where the feedforward model compensation is $v_{3 a}=-\psi_{3}$ and the feedback stabilization term $v_{3 s}=-k_{3 s 1} z_{3}$. Thus we have

$$
\dot{z}_{3}=-k_{3} z_{3}+z_{4}, \quad k_{3}=k_{3 s 1} .
$$

\subsection{Step 4-Flux Inner Loop Control Design}

The derivative of the virtue control discrepancy is

$$
\dot{z}_{4}=v_{4}+\psi_{4}+\tilde{d}_{4},
$$


where

$$
\begin{aligned}
\psi_{4} & =2 a_{10} a_{5}\left(\hat{x}_{4}^{2}+\hat{x}_{5}^{2}\right)-2 a_{10} a_{7}\left(\hat{x}_{2} \hat{x}_{4}+\hat{x}_{3} \hat{x}_{5}\right) \\
& +2 a_{10}\left(l_{21} \tilde{x}_{2} \hat{x}_{4}+l_{22} \tilde{x}_{3} \hat{x}_{4}+l_{31} \tilde{x}_{2} \hat{x}_{5}+l_{32} \tilde{x}_{3} \hat{x}_{5}\right) \\
& +2\left(l_{41} \hat{x}_{4}+l_{51} \hat{x}_{5}\right)\left(-\left(a_{7}+l_{21}\right) \tilde{x}_{2}-l_{22} \tilde{x}_{3}\right) \\
& +2\left(l_{42} \hat{x}_{4}+l_{52} \hat{x}_{5}\right)\left(-l_{31} \tilde{x}_{2}-\left(a_{7}+l_{32}\right) \tilde{x}_{3}\right) \\
& +2\left(l_{41} \tilde{x}_{2}+l_{42} \tilde{x}_{3}+a_{10} \hat{x}_{2}-2 a_{8} \hat{x}_{4}\right) \\
& \times\left(-a_{8} \hat{x}_{4}-a_{9} \hat{x}_{1} \hat{x}_{5}+a_{10} \hat{x}_{2}\right) \\
& +2\left(l_{41} \tilde{x}_{2}+l_{42} \tilde{x}_{3}+a_{10} \hat{x}_{2}-2 a_{8} \hat{x}_{4}\right)\left(l_{41} \tilde{x}_{2}+l_{42} \tilde{x}_{3}\right) \\
& +2\left(l_{51} \tilde{x}_{2}+l_{52} \tilde{x}_{3}+a_{10} \hat{x}_{3}-2 a_{8} \hat{x}_{5}\right) \\
& \times\left(-a_{8} \hat{x}_{5}+a_{9} \hat{x}_{1} \hat{x}_{4}+a_{10} \hat{x}_{3}\right) \\
& +2\left(l_{51} \tilde{x}_{2}+l_{52} \tilde{x}_{3}+a_{10} \hat{x}_{3}-2 a_{8} \hat{x}_{5}\right)\left(l_{51} \tilde{x}_{2}+l_{52} \tilde{x}_{3}\right) \\
& +k_{3}\left(-k_{3} z_{3}+z_{4}\right)-\ddot{\psi}_{d} \\
\tilde{d}_{4} & =\left(2 a_{5} l_{41} \hat{x}_{4}-2 a_{6} l_{42} \hat{x}_{4} \hat{x}_{1}+2 a_{5} l_{51} \hat{x}_{5}-2 a_{6} l_{52} \hat{x}_{5} \hat{x}_{1}\right) \tilde{x}_{4} \\
& +\left(2 l_{41} a_{6} \hat{x}_{1} \hat{x}_{4}+2 l_{51} a_{6} \hat{x}_{1} \hat{x}_{5}+2 l_{42} a_{5} \hat{x}_{4}+2 l_{52} a_{5} \hat{x}_{5}\right) \tilde{x}_{5} \\
& +2\left(l_{41} \hat{x}_{4}+l_{51} \hat{x}_{5}\right) a_{6} \tilde{x}_{1} x_{5}-2\left(l_{42} \hat{x}_{4}+l_{52} \hat{x}_{5}\right) a_{6} \tilde{x}_{1} x_{4} .
\end{aligned}
$$

Note $\tilde{d}_{4}$ is the disturbance due to state estimation errors, and

$$
\begin{aligned}
& v_{4}=a_{21} b u_{1}+a_{22} b u_{2}, \\
& a_{41}=2 a_{10} \hat{x}_{4}, \\
& a_{42}=2 a_{10} \hat{x}_{5} .
\end{aligned}
$$

Design control input as

$$
v_{4}=v_{4 a}+v_{4 s}
$$

where the feedforward model compensation is

$$
v_{4 a}=-\psi_{4}-z_{3},
$$

and the feedback stabilization control term consists of the nominal stabilization term $v_{4 s 1}$ and the robust control term $v_{4 s 2}$,

$$
\begin{aligned}
& v_{4 s}=v_{4 s 1}+v_{4 s 2}, \\
& v_{4 s 1}=-k_{4} z_{4} .
\end{aligned}
$$

Thus

$$
\dot{z}_{4}=-k_{4} z_{4}+v_{4 s 2}+\tilde{d}_{4}-z_{3} .
$$

From the assumption of the boundedness of state estimation error, there exists a $v_{4 s 2}$ such that

$$
\begin{aligned}
& \text { - } z_{4}\left(v_{4 s 2}+\tilde{d}_{4}\right) \leq \epsilon_{4} \text {, } \\
& \text { - } z_{4} v_{4 s 2} \leq 0 \text {, }
\end{aligned}
$$

where $\epsilon_{4}$ is a design parameter which can be arbitrarily small. One example of $v_{4 s 2}$ that satisfying above conditions is:

$$
v_{4 s 2}=-\frac{1}{4 \epsilon_{4}} h_{4}^{2} z_{4},
$$

where $h_{4}=\delta_{d_{4}}$ and $\delta_{d_{4}}$ is the bound of $\tilde{d}_{4}$.

Remark 4.4 The control input can be determined by

$$
\left[\begin{array}{l}
u_{1} \\
u_{2}
\end{array}\right]=\frac{1}{b}\left[\begin{array}{cc}
-a_{1} \hat{x}_{5} & a_{1} \hat{x}_{4} \\
2 a_{10} \hat{x}_{4} & 2 a_{10} \hat{x}_{5}
\end{array}\right]^{-1}\left[\begin{array}{l}
v_{2 a}+v_{2 s} \\
v_{4 a}+v_{4 s}
\end{array}\right] .
$$

For control input voltage to has unique solution, we have

$$
\left|\begin{array}{cc}
-a_{1} \hat{x}_{5} & a_{1} \hat{x}_{4} \\
2 a_{10} \hat{x}_{4} & 2 a_{10} \hat{x}_{5}
\end{array}\right|=-2 a_{1} a_{10}\left(\hat{x}_{4}^{2}+\hat{x}_{5}^{2}\right) \neq 0,
$$

which means flux modulus estimate should not be zero.

\subsection{Stability Analysis}

Remark 4.5 Control $v_{3}$ and $v_{4}$ guarantees the boundedness of $z_{3}$ and $z_{4}$. Similar to the Remark 4.3, taking a Lyapunov function candidate

$$
V_{s 4}=\frac{1}{2} z_{3}^{2}+\frac{1}{2} z_{4}^{2}
$$

and computing its time derivative, one can derive

$$
V_{s 4} \leq \exp \left(-\lambda_{4} t\right) V_{s 4}(0)+\frac{\epsilon_{4}}{\lambda_{4}}\left[1-\exp \left(-\lambda_{4} t\right)\right],
$$

with $\lambda_{4}=2 \min \left(k_{3}, k_{4}\right)$. This implies the boundedness of $z_{3}$ and $z_{4}$. If further assuming $z_{3}$ and $z_{4}$ dynamics are only subject to parametric uncertainties (i.e., $\tilde{d}_{4}=0$ ), the zero solution of $z_{3}$ and $z_{4}$ dynamics is asymptotically stable, i.e., $z_{3}, z_{4} \rightarrow 0$, as $t \rightarrow \infty$.

To analyze the stability of the entire closed-loop system, we first define a Lyapunov function candidate

$$
V_{s}=V_{s 2}+V_{s 4}
$$

Theorem 4.6 Given Assumption 4.2, the closed-loop system (2) with a state estimator (4) and the control $u$ (54) has bounded states, and the Lyapunov function candidate $V_{s}$ in (58) is bounded by

$$
V_{s} \leq \exp (-\lambda t) V_{s}(0)+\frac{\epsilon}{\lambda}[1-\exp (-\lambda t)],
$$

with $\lambda=2 \min \left(k_{1}, k_{2}, k_{3}, k_{4}\right)$.

Proof: Given the Lyapunov function candidate $V_{s}$ and Assumption 4.2, we have

$$
\begin{aligned}
\dot{V}_{s} & =-k_{1} z_{1}^{2}-k_{2} z_{2}^{2}-k_{3} z_{3}^{2}-k_{4} z_{4}^{2} \\
& +z_{2}\left(v_{2 s 2}+\tilde{d}_{2}\right)+z_{4}\left(v_{4 s 2}+\tilde{d}_{4}\right) \\
& \leq-k_{1} z_{1}^{2}-k_{2} z_{2}^{2}-k_{3} z_{3}^{2}-k_{4} z_{4}^{2}+\epsilon_{2}+\epsilon_{4} \\
& \leq-\lambda V_{s}+\epsilon,
\end{aligned}
$$

which implies (59) and the boundedness of all states. This completes the proof.

\section{Simulation Results}

In this section, we present simulation results for the proposed speed sensorless control algorithm which includes the $\mathrm{EKF}$ as an state observer. The goal is to demonstrate the following:

1) the proposed method can achieve high bandwidth $(200 \mathrm{~Hz})$ and high precision speed sensorless tracking control of a typical induction motor under practical implementation constraints $(20 \mathrm{KHz}$ sampling rate and $400 \mathrm{~V}$ input saturation) with only stator voltage and current measurements;

2) the gain selection for the proposed method is straightforward;

3) the proposed method can track various trajectories as long as the state and control input constraints are considered. No dependence of controller and/or observer on the trajectory is found.

The simulation is implemented in Matlab with sampling rate of $20 \mathrm{KHz}$ and the following induction motor parameters [2] $R_{s}=2.3 \Omega, R_{r}=4.95 \Omega, L_{m}=0.523 H, L_{s}=$ 
$0.538 H, L_{r}=0.5396 H, J=0.02 \mathrm{~kg} \cdot \mathrm{m}^{2}, b 1=0.001$, $n p=2$, and $T_{f}=0.50$.

To achieve the control bandwidth of $200 \mathrm{~Hz}$, the control gains are selected as $k_{1}=k_{2}=k_{3}=k_{4}=1200$, and no further gain tuning is necessary. As a result, there is no fundamental limitation on the achievable control bandwidth, as long as the control input is not severely saturated before the observer, which is independent from the controller, converges. The desired flux modulus is $\phi_{d}=0.5$ for all cases.

The initial condition for the states are $x=$ $\left[\omega_{d}(0), 2,2,0.5,0.5\right]^{T}$, where $\omega_{d}(0)$ is the desired speed at $t=0$. The control inputs has a saturation limit of $400 \mathrm{~V}$. The observer has initial estimation errors of 10 percent. For implementation of EKF, the current measurements are assumed to have noises of $2 \mathrm{~mA}$ and the process noises are all assumed to be $0.001 \times T_{s}$.

We tested the proposed observer-controller by tracking different trajectories. Fig. 2 shows the high-bandwidth tracking of $200 \mathrm{~Hz}$ sinusoidal trajectory. After the short transient for the observer to converge, the controller tracks the speed reference signal closely. Fig. 3 shows the simulations result of tracking a step reference speed signal, which is first filtered by a second order critically damped transfer function with $200 \mathrm{~Hz}$ natural frequency. The tracking transient shows small settling time close to $1 / 200$ second. Fig. 4 further demonstrates the excellent tracking performance for a ramp up and down speed reference trajectory. The controller performs consistently well for all tested trajectories, and the closed-loop system performance appears to be independent of the reference trajectory.
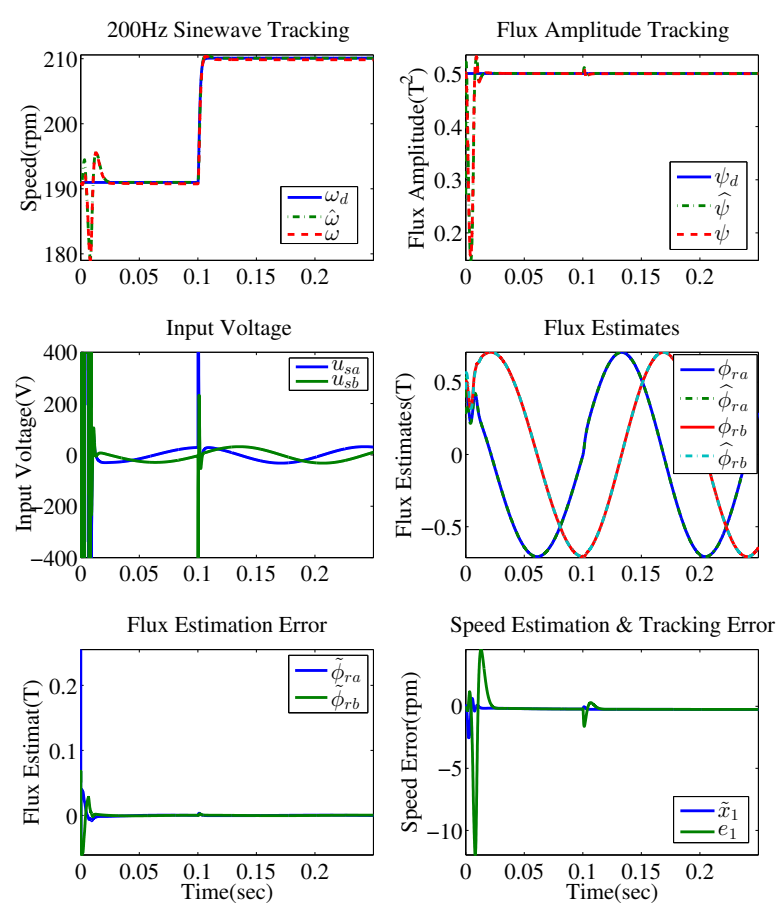

Fig. 3: Tracking a step reference with $0.005 \mathrm{sec}(200 \mathrm{~Hz})$ settling time.
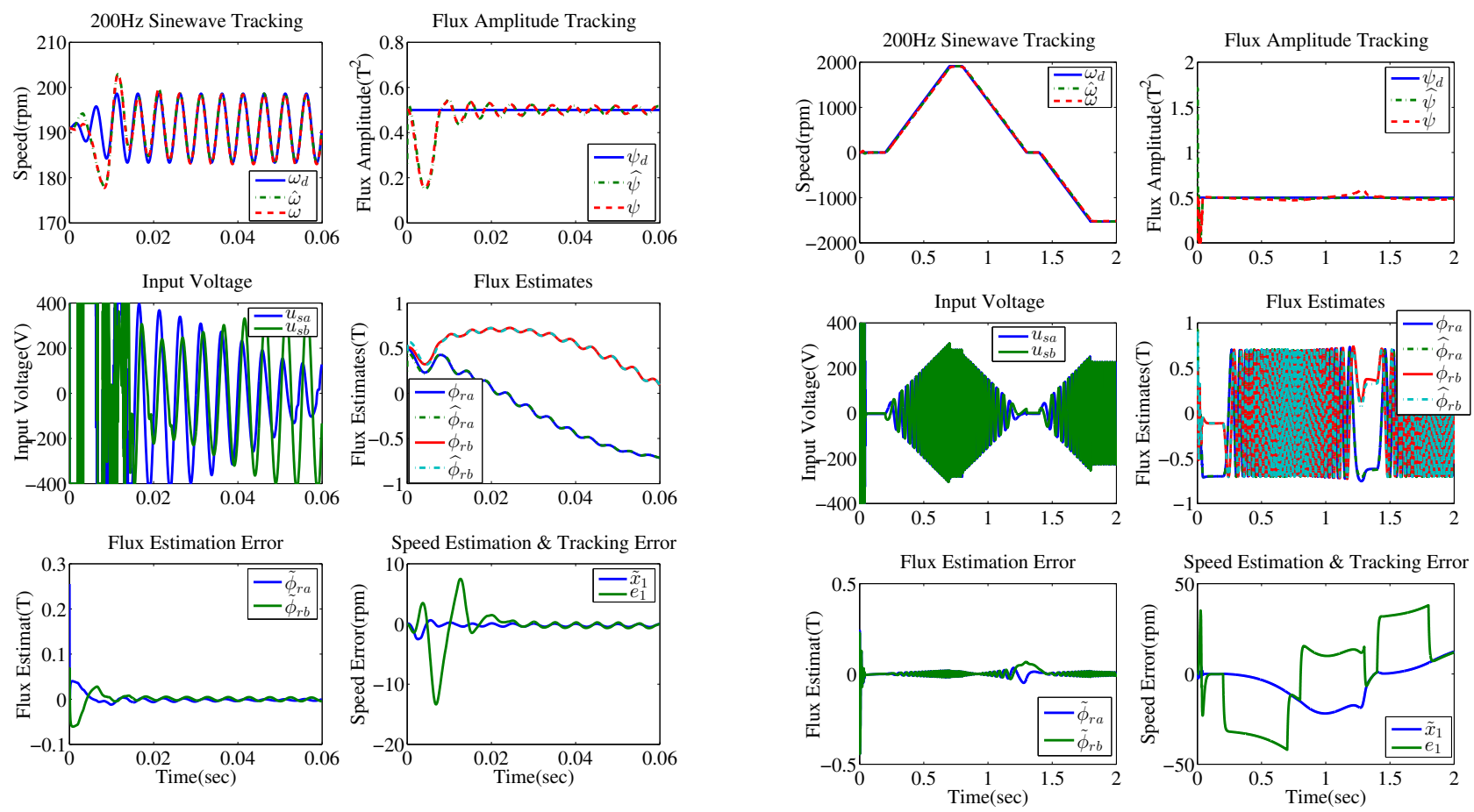

Fig. 4: Tracking a ramp reference trajectory. 


\section{Conclusion and Future Work}

In this paper, we proposed an integrated control and observer design framework for speed sensorless control of induction motors with only current measurements. With a general observer structure, the backstepping-based robust control design explicitly considers all the state estimation and tracking errors. Simulation results shown that the proposed method leads to high bandwidth and high precision speed sensorless tracking control of a typical induction motor under practical implementation constraints, in contrast to the severe bandwidth and performance limitations inherent to previous methods. Ongoing work includes experimental verification of the proposed method.

\section{References}

[1] M. Montanari, S. M. Peresada, C. Rossi, and A. Tilli, "Speed sensorless control of induction motors based on a reducedorder adaptive observer," vol. 15, no. 6, pp. 1049-1064, 2007.

[2] H. K. Khalil, E. G. Strangas, and S. Jurkovic, "Speed observer and reduced nonlinear model for sensorless control of induction motors," Control Systems Technology, IEEE Transactions on, vol. 17, no. 2, pp. 327-339, 2009.

[3] R. Marino, P. Tomei, and C. M. Verrelli, Induction Motor Control Design. London, UK: Springer, 2010.

[4] J. Chiasson, "Dynamic feedback linearization of the induction motor," vol. 38, no. 10, pp. 1588-1594, 1993.

[5] L. Harnefors, "Globally stable speed-adaptive observers for sensorless induction motor drives," vol. 54, no. 2, pp. 12431245, Apr. 2007.

[6] D. J. Atkinson, P. P. Acarnley, and J. W. Finch, "Observers for induction motor state and parameter estimation," vol. 27, no. 6, pp. 1119-1127, Nov./Dec. 1991.

[7] C. Schauder, "Adaptive speed identification for vector control of induction motors without rotational transducers," vol. 28, no. 5, pp. 1054-1061, Sep./Oct. 1992.

[8] H. Kubota, K. Matsuse, and T. Nakano, "DSP-based speed adaptive flux observer of induction motor," vol. 29, no. 2, pp. 344-348, Mar./Apr. 1993.

[9] H. Kubota and K. Matsuse, "Speed sensorless field-oriented control of induction motor with rotor resistance adaption," vol. 30, no. 5, pp. 1219-1224, Sep./Oct. 1994.

[10] K. Ohyama, G. M. Asher, and M. Sumner, "Comparative analysis of experimental performance and stability of sensorless induction motor drives," vol. 53, no. 1, pp. 178-186, Feb. 2006.

[11] C. Lascu, I. Boldea, and F. Blaabjerg, "A class of speedsensorless sliding-mode observers for high-performance induction motor drives," vol. 56, no. 9, pp. 3394-3403, Sep. 2009.

[12] M. Ghanes and G. Zheng, "On sensorless induction motor drives: sliding-mode observer and output feedback controller," vol. 56, no. 9, pp. 3404-3413, Sep. 2009.

[13] S. Solvar, B. Le, M. Ghanes, J. P. Barbot, and G. Santomenna, "Sensorless second order sliding mode observer for induction motor," in IEEE International Conference on Control Applications, Yokohama, Japan, 2010, pp. 1933-1938.

[14] M. Hilairet, F. Auger, and E. Berthelot, "Speed and rotor flux estimation of induction machinese using a two-stage extended kalman filter," vol. 45, no. 8, pp. 1819-1827, Aug. 2009.

[15] W. Leonhard, Control of Electrical Drives. Springer, 2001.

[16] B. Yao and M. Tomizuka, "Adaptive robust control of siso nonlinear systems in a semi-strict feedback form," Automatica, vol. 33, no. 5, pp. 893-900, 1997. 